\title{
Construction of Geographical Information Collection System Based on Wireless Sensor Network
}

\author{
Yan Jiang \\ Department of Information Technology, Inner Mongolia Business and Trade Vocational College, \\ Hohhot, 010070, China
}

Keywords: Wireless sensor networks geographical information, Collection system, Construction

\begin{abstract}
. with fast development of science and technology, wireless sensor network has applied in large amount due to its flexible installation and convenient group features. It's applied for security detection in areas such as intelligent buildings, military, and large industrial Orchard. Arrange large numbers of cheap micro sensor node in monitoring range, use wireless network for communications to form a shared network, to achieve the purpose of collection and monitoring of geographic information. This paper analyzes the construction of wireless sensor network of geographical information collection system.
\end{abstract}

\section{Introduction}

Wireless sensor network mainly sets a lot of cheap micro-node sensors within the region and use wireless communications to set a variety of automatic organization system, which is used to gather, perceive and handle physical information within the network coverage area. At this stage, sensors can be used to get information of the whole physical world, for example, explosions, sound, pressure, temperature, vibration, humidity, etc., Then set up corresponding sensor networks, matching organically with thousands of space and sensor nodes, forming joint space-time detection system. Because of its characteristics, such as flexible layout, multiple functions, interconnection of different sensors to form multiple network topology, wireless sensors have a very rich application value in wireless sensor networks, therefore, ever since the development of wireless sensor networks, it's subject to controls of various institutions, its importance is equivalent to Internet, Internet information main connects information space, and wireless sensor networks can be connected to physical space.

\section{Construction of wireless sensor networks for geographic information collection}

With constant application and development of wireless access technology and wireless network technologies, in network organizations, whether a wireless LAN or a radiotelephone system, is basically traditional Multihop routing and cellular structure. Using a cellular wireless network structure to build a system, would have features such as convenient management, simple structure and mature technology, but when applying it in geographical information collection system in large-scale, there will still be some defects and problems. Geographical information collection system established on wireless communications network is a special system which has higher requirements for communication range, signal processing and power consumption, in addition, because of its properties of variability of the network and dynamic nodes location, traditional fixed cellular base station can no longer meet the actual demand.

Wireless sensor network system consists of two categories, aggregation nodes and sensor nodes. Power wireless transmitting nodes with battery; deploy randomly in the detection area, use self-organization structure to build network. Sensor nodes would send interested event with multi-hop methods to the Sink. In the process of transferring data, the sensor most likely are combined with related nodes, reducing network load to a certain extent and increase the upload information database. 
In applications, wireless sensor network can be used as nerve endings networks, it is an important media network for liaison and communication between the physical world and the human world, and it has the advantages of extensiveness and long-term unattended operation. The nodes has simple and cheap design, typically, there are 4 modules, namely, process modules, sensor modules, power supply module and wireless communication modules. Sensor module is used to capture and convert data within the detection area; sensor module is used to control the entire bus system operation; wireless communication module is used to connect to other nodes, collect and exchange data; power supply module is used to provide energy for sensor node, they are generally micro-batteries.In the process of development and research of wireless sensor network, it would be affected or strained by data transmission, data storage, energy supply, system stability and network synchronization. To add new function effectively to the system, we need to constantly optimize wireless network resources management and wireless sensor network performance. Resources management main includes the following aspects: process management, sensor management, network control and network planning, it can reduce network energy consumption and improve network performance and network of stability. Fusion information mainly has following several aspects: data fusion, sensor fusion, wave tracking and estimation, data mining, and so on, it can, to some extent, increase capacity to collaboration process and network information transmission.

This paper mainly analyze specification of geographic information collection system and actual application, using layered network structure to ensure established wireless sensor network framework would meet national development situation, above framework is divided into Sink aggregation node layer, public network access layer and peripheral band sensor node detection layer, to ensure that we can form three layer autonomy group network system, developing direction of this system is different from existing Zigbee, this system is sat up according to the actual situation in line with the national development of wireless sensor networks. Zigbee standards have better network performance, but it still has weak points such as weak diffraction performance and short distance transmission which makes it unable to connect to backbone network and group in the villages, towns, wild places, therefore, it cannot be applied widely. After analysis of China's basic national conditions, we do need long-distance transmission and a strong ability to diffraction for domestic networks construction [1].

Bottom end of the sensor network can be called micro-grid, it is short distance wireless sensor network with low speed, low power consumption and do not support local moves. Sink aggregation node in the middle layer is called the middle-band network, it has high rate and support random access, random layout, random extension, long distance transmission, and local moves.This mid-level networks can solve problems like limited storage, sensor network energy, process capacity and bandwidth. We can use the above system structure to arrange proper geographical data location in the city, set collection node at each intersection, and can automatically form into strip network, arrange main information access points along the main road.With the constant construction and development of information city, we can use complete fiber optic network to achieve the purpose of quick coverage ensuring that it can adapt to different applications locations and geographic conditions to transmit information in real time, it can also process existing geographic monitoring information and testing data, and publish results on the public network.

\section{Build hardware platform for the system}

Wireless magnetic sensor node consists of four parts, wireless communication modules, microprocessors, sensors, power supply module.

\section{Sensor}

We can use the sensor to detect physical quantities in the real world, the sensor can provide some data for geographical monitor, such as humidity, temperature, light, sound, vibration, smoke, organic etc. organic collection of these sensors so as to shape a digital geographic information composite figure. We can use magnetic induction to detect ferrous material in the car chassis. This kind of 
magnetic induction can be reasonably applied to control ramp, testing data, management of parking spaces, and so on. The Earth's magnetic field is about 0.5 Gauss, so, sensor with high sensitivity and low magnetic intensity is needed to properly detect distortions. In all sensors, Magnet resistive sensor can meet criteria of low energy and volume space features; it can also meet the needs of low intensity test requirements [1].

\section{AMR sensors}

This kind of sensor is best suited for work in the vicinity of the Earth's magnetic field. Magneto-resistive sensors can be divided into giant magneto-resistance sensor GMR and anisotropic magneto-resistive sensors AMR. AMR sensor has a certain direction, and can provide consistent sensitive axis and magnetic field strength amplitudes, but GMR does not have these features, it can only provide one-dimensional numerical information, it is a Wheatstone bridge which can not only detect the direction and strength of the magnetic field, but also DC static magnetic fields.

\section{Honeywell HMC1051Z}

This paper studies AMR magneto resistive sensor based on wireless sensor network node, which belongs to the Honeywell HMC1051Z, it has the advantage of small size, high sensitivity and low power consumption. But the biggest drawback is in fact that if we want to guarantee high sensitivity, we need to provide periodic Wheatstone bridge which will not only restrict the selection of batteries, it will increase power consumption. Therefore, it is not very suitable for design and delivery of large currents.

\section{Communication protocol}

Wireless communications will in some degree affect sensor node cycle, after test we can find that about $90 \%$ of energy after test can be used for wireless receiving module, in low power module, energy consumption of signal launches and signal receive are basically the same, so, in the process of communications agreement design, we need to full consider interval between receive and launches to minimize energy consumption to maximize life cycle of sensor. Increase of communication distance will reduce the strength of receiving signal. Communications range is also limited by the antenna gain, sensitivity and efficiency. Typical sensitivity of the receiver is between 085 110dbm [3]. When using $0 \mathrm{dBm}$ to launch, calculate receiver sensitivity according to $-85 \mathrm{dBm}$ in free outdoor environment, 25 50m transmission distance can be achieved, when maximizing system cycle as well as designing the network nodes, rational use of wireless configuration can play a facilitating role.

\section{Select battery}

Wireless sensor networks cycle is closely related to power. Limited energy is the most important factor while designing micro-embedded sensors; in addition, reducing the size of micro-sensors and processors has gone far beyond the battery, resulting in increased battery cost in wireless sensor networks. Further maintenance or replacing batteries can find that these factors are unable to cater for the actual demand for running; therefore, in the design process, power supply need to be fully considered.As for control of wireless sensor networks node, important features includes hibernation mode. Micro-controller node is dormant $99.9 \%$ of the time, so waken time and sleep power consumption is the most important factor. For different types of controllers, hibernation quiescent current is in 1 50A, wake up in the $6 \mathrm{~s} \sim 10 \mathrm{~ms}$ [4]. A shorter wakeup time can switch into hibernation mode faster; proper application of response sensor nodes with high frequency if sleep mode power consumption is not increased. Zinc batteries have high energy density, but also have rapid leakage problems; therefore, it's suitable for applications in short-run case. Lithium battery has features of fast charging memory and high energy density, but it needs long time to recharge; polymer battery is flexible with high energy density, but it's expensive. Fuel cells are a new type of energy with a lot of attention.

\section{Physical platform and the microprocessor}

Microprocessors can control electronics processors, microprocessors for wireless

on a computer, compared with general-purpose sensor networks are in low cost and can 
self-regulate.Memory, processing units, peripherals, digital I/O interfaces as well as digital analog converter can be integrated on an integrated circuit, in integrated way, we can be reduce chip count, reduce PCB area and omit wiring. Many sensor nodes use digital signal processor DSP, which can be used as micro-controller.Starting from sampling analog circuits, form conversion, the whole process needs to have wireless communications and data processing. Unprocessed full range includes peripheral circuit as well as MSP430F1611 microcontroller; they can control and calculate the circuit.MSP430F1611 MCU has advantages such as low power consumption, speedy calculation. At high speed, the clock rate can reach $8 \mathrm{MHz}$, at low speed, it can reach $32768 \mathrm{MHz}$, interface SPI port 0: SPI port are required in order to properly configure the SPI port and AD8403, you can achieve the objective of rational communication in a wireless communication module. In times of low-power consumption, the microprocessor needs to appropriately compare output pulse for collecting geographic information, which can wake up single-chip to run the digital-analog converter module, so that you can continue to collect the output signal amplitude; after GIS data collection when below the set value and stability voltage value, single-chip microcomputer can resume at low power again [5].

\section{Concluding remarks}

In summary, when building wireless sensor network for geographic information collection system, we need to build wireless sensing network system, and consider method for effective collection of geographic information, reasonable selection of sensor signal and establishment of logistics hardware platform, distance change will increase energy consumption, damage base station node, cause system paralysis, to this, we need to combine reality for reasonable configuration of wireless network, our proposal of wireless sensor network for geographic information collection network use layered network structure, Reasonable choice of battery, physics platform, microprocessor and so on, which provide the basis for future development.

\section{References}

[1] Zhang zenglin, Yu Xiao Qing. Soil Data Acquisition System Based on Wireless Sensor Networks. Water-saving Irrigation, 2011 (12): 41-43,49.

[2] Tan Yuling. Temperature and Humidity Data Acquisition System Based on Wireless Sensor Network . Communications World, 2015 (4): 215-215.

[3] Tan Weihan, Wang Weixing, xiaoguokun etc. Tea Information Acquisition System Design Dased on Wireless Sensor Network. Agricultural Network Information, 2010 (7): 95-98.

[4] Liu Ji, Xu Qun. ZigBee Wireless Sensor Network Technology Applicationin IN Oil Field Information Collection System. Electrical Design Engineering, 2014,22 (5): 157-160.

[5] Xu Lifeng.Traffic Information Acquisition System Based on Wireless Sensor Network Applications. Computer Applications and Software, 2012,29 (4): 236-241,262. 